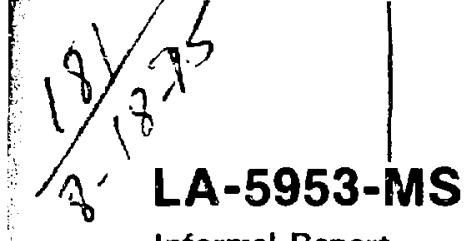

Informal Report
UC-38

Reporting Date: April 1975 Issued: June 1975

\title{
Design of a Force-Free Inductive Storage Coil
}

\author{
by
}

Osman K. Mawardi*

-Present address: Case Western Reserve University,

School of Engineering, Cleveland, $\mathrm{OH} 4: 1106$.

An Affirmative Action/Equal Opportunity Emgleyer 
In the interest of prompt distribution, this report was not edited by the Technical Information staff.

Work supported by US Energy Research and Development Administration, Division of Energy Storage.

Printed in the Uatted States of America. Available from National Technical Information Service

US Department of Commerce

5285 Port Roycl Road

Springfield, VA 22151

Price: Printed Copy \$4.00 Microfiche \$2.25

This repart was prepored as an accoust of werk sponsored

by the United Sictes Government. Neither the Uniled States

nor the United Stales Enerky Reacarch and Development Ad.

tractors. unbcontractors, or their employees. makes ony

warranty, express or implied, or asumes any lecalliebiltev or

responsibility for the accuracy, completeness. or usefulness of

any information. eppartulus, product, or process disclosed, of

reprearn
rixht. 
DESIGN OF A FORCE-FREE INDUCTIVE STORAGE COIL

by

Osman K. Mawardi

\section{ABSTRACT}

Force-free coils have been considered for various applications as energy storage devices. A novel energy storage system consisting of both toroidal and poloidal coils has been developed. In this system, the Lorent $z$ forces on the two coils are in opposition and can be made to cancel by having the proper ratio of ampere-turns in the two coils. A general discussion of force-free coil design is given, and the toroidal-poloidal coil system is described. The materials costs of magnets constructed with this and other geometries are compared. The conclusions are that conductor cost of this system lies between those of solenoids and toroids, and that the costs of structural material may be considerably less than either of these simpler coils.

\section{INTRODUCTION}

The application of inductive storage by means of superconducting coils to peak load shaving and load leveling in an electric power system has been studied by many workers in the field. It has been pointed out, ${ }^{2}$ however, that the mechanical structure needed to counterbalance the electromechanical forces in inductors accounts for a major fraction of the overall cost of the inductive storage installation. Hence, idéntifying a practical force-free configuation may lead to a substantial saving in cost.

Although the theory of force-free coils, coils in which the current and field are parallel, $\underline{\mathrm{J}}=\underline{\mathrm{H}}$ has been known for some time, ${ }^{3}$ there has been some confusion surrounding practical implications of these coils. It is important to realize, for instance, that a coil of finite size cannot be strictly forcefree. This fact, which was pointed out for the first time by Rosenbluth, is described in detail in Longmire's text, "Elementary Plasma Physics." 5 The proof makes use of well-known arguments based. on Maxwell's development of the stress tensor associated with the electromagnetic field and on an extension of the virial theorem of Clausiu-

For convenience, a brief outline of Longmire's proof has been duplicated in the appendix. The essential conclusions are given below.

1) When a system consisting of a coil and its associated magnetic field is in equilibrium, then the mechanical energy due to the electromechanical stresses in the coil cannot be less than the magnetic energy of the field.

2) The mechanical energy in the coil is a minimum when the coil is force-free.

3) The stresses in a force-free coil are tensile in character. In a conventional (nonforcefree) coil, the forces are both tensile and compressive.

4) The mass of a coil - designed so that the material of the coil is subjected to the maximum allowable working stresses - is directly proportional. to the stress energy in the coil. An immediate corollary of this conclusion is that a force-free coil will lead to a structure with the minimum mass. In order to illustrate this statement, Stekly ${ }^{2}$ calculated the mass of a structure of a conventional turoidal coil in which the winding is purely poloidal. He subsequently pointed out that such a coill has a mass three times that of a force-free toroidal coil associated with the same magnetic energy.

The standard procedure of estimating force-free fields is to look for current distributions in which 
the current is everywhere parallel to the magnetic field. This is the approach that has been followed here. However, an additional requirement has been int roduced, viz., that only solutions leading to surface currents which can be easily produced in practice are selected.

\section{FORMULATION OF DESIGN PROCEDURE}

The starting point of the calculations is the basic equation for force-free criteria,

$\underline{F}=\underline{J} \times \underline{H}=(\nabla \times \underline{H}) \times \underline{H}=0$.

As is well known, the above expression implies that the current is parallel to the field,

$(\nabla \times \underline{H})=\alpha \underline{H}=\underline{J}$,

where $\alpha$ is a function of position which needs to be evaluated.

It is convenient to introduce a vector potential A, defined by

$\underline{H}=(\nabla \times \underline{A})$.

Combining the above expression with Ampère's equation,

$\underline{J}=(\nabla \times \underline{H})$

$=(\nabla \times \nabla \times \underline{A})$.

then the set of Eqs. 2, 3, and 4 completely defines the problem, once the shape of the coil is decided upon.

It is proposed to discuss the toroidal configuration. Conventional toroidal coils for which the field is completely inside the torus have the disadvantage of displaying a poor figure of merit, ${ }^{6}$ defined as the magnetic energy stored per unit mass of conductors. This statement is not for force-free coils in which the field produced by the tisroidal coil extends to all space. The stored energy increases appreciably and it will be shown that the figure of merit improves a great deal.

An axi-symetrical magnetic field which is independent of the azimuthal component can be expressed by: ${ }^{7}$
$\left.\underline{H}=\underline{i}_{2} \times \underline{r}^{T}+\nabla \times \underline{i}_{2} \times \underline{r} P\right)$

where $T$ and $P$ are toroidal and poloidal components, respectively, and $\underline{i}_{z}$ is a unit vector along $z$, the axis of symmetry. It is convenient to express the magnetic field in cylindrical cocrdinates: $2, \tilde{w}$, and $\phi$, so that

$\underline{H}=-\tilde{\omega} \frac{\partial P}{\partial z} \underline{\underline{j}}_{\tilde{\omega}}+\tilde{\omega} \mathrm{T} \underline{\underline{i}}_{\Phi}+\frac{1}{\dot{\omega}} \frac{\partial}{\partial \tilde{\omega}}\left(\tilde{\omega}^{2} \mathrm{P}\right) \underline{\underline{i}}_{z}$,

where $\underline{i}_{\tilde{w}}, \underline{\underline{i}}_{\phi}$ are unic vectors along the radial and azimuthal directions.

In a similar manner, we define the vector potential in eerms of toroidal and poloidal components $T^{\star}$ and $P^{*}$ respectively. Thcrefore one finds:

$\underline{A}=\underline{i}_{2} \times \underline{r} T^{*}+\nabla \times\left(\underline{i}_{2} \times \underline{r}^{p^{*}}\right)$.

The components of the magnetic field are found from

$\underline{H}=(\nabla \times \underline{A})=\tilde{\omega} \frac{\partial T^{\star}}{\partial z} \underline{i}_{\tilde{\omega}}-\tilde{\omega} \omega_{5} P^{\star} \underline{-}_{\phi}+\frac{1}{\tilde{\omega}} \frac{\partial}{\partial \tilde{\omega}}\left(\tilde{\omega}^{2} T^{\star}\right) \underline{i}_{z}$,

where

$\Delta_{5} \equiv \frac{\partial^{2}}{\partial \tilde{\omega}^{2}}+\frac{3}{\tilde{\omega}} \frac{\partial}{\partial \tilde{\omega}}+\frac{\partial^{2}}{\partial z^{2}}$.

The requirement of force-free fields together with Eq. 8 yields

$j_{\tilde{\omega}}=\alpha H_{\tilde{\omega}}=\frac{\partial}{\partial z}\left(\tilde{\omega} \Delta_{5} P^{*}\right)=-\alpha \tilde{\omega} \frac{\partial T^{*}}{\partial z}$,

$j_{\phi}=\alpha H_{\phi}=-\left(\frac{1}{\tilde{\omega}} \frac{\partial^{2}\left(\tilde{\omega}^{2} T^{\star}\right)}{\partial z^{2}}+\frac{\partial}{\partial \omega} \frac{1}{\tilde{\omega}} \frac{\partial}{\partial \tilde{\omega}^{2}}\left(\tilde{\omega}^{2} T^{\star}\right)\right)=$

$-\alpha\left(\tilde{\omega} \Delta_{5} p^{\star}\right)$,

$j_{z}=\alpha H_{z}=-\frac{1}{\tilde{\omega}} \frac{\partial}{\partial \tilde{\omega}}\left(\tilde{\omega}^{2} \Delta_{5} P^{*}\right)=\alpha \frac{1}{\tilde{\omega}} \frac{\partial}{\partial \tilde{\omega}^{*}}\left(\tilde{\omega}^{2} T^{*}\right)$.

An immediate consequence of Eq. 10, which we rewrite as

$\frac{\partial^{2}}{\partial z^{2}}\left(\tilde{\omega}^{2} T^{*}\right)+\tilde{\omega} \frac{\partial}{\partial \tilde{\omega}} \frac{1}{\tilde{\omega}} \frac{\partial}{\partial \tilde{\omega}}\left(\tilde{\omega}^{2} T^{*}\right)=-\tilde{\omega} j_{\phi}$.

is that any $\left(\tilde{u} j_{\phi}\right)=f n\left(\tilde{\omega}^{2} T^{*}\right)$ which satisfies Eq. $10^{\prime}$ describes a force-free configuration. This important 


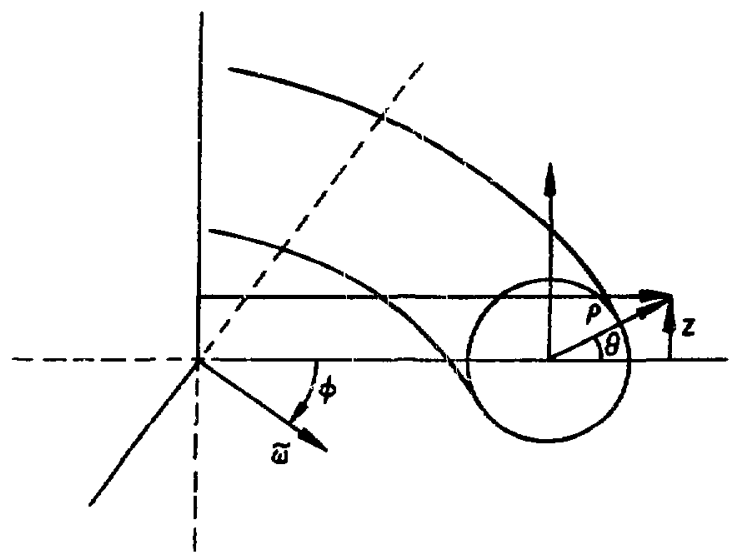

Fig. 1. Coordinate system used for calculations of toroidal coil geometries. $\tilde{\omega}, d$, and $z$ are the usual polar coordinates. $\rho$ and $\theta$ are the poloidal coordinates.

result was pointed out by Furth et al (Ref. 4). To completely define the problem we need to find the component $\mathrm{P}^{*}$ and the function $\alpha$. We will find it very useful to introduce polar coordinates $0, \theta$, as shown in Fig. 1, referred to a plane which includes the axis of symmetry of the toroid. If we introduce a poloidal current $j_{\theta}$, ther:

$i_{\tilde{\omega}}=-\sin \theta \mathbf{j}_{\theta}$,

and

$j_{z}=\cos \theta j_{\theta}$

Multiplying Eq. 9 by $\sin \theta$, Eq. 10 by $\cos \theta$, and subtracting one from other, we obtain

$\sin \theta \frac{\partial}{\partial z}\left(\tilde{\omega}^{2} \Delta_{5} P^{*}\right)+\cos \theta \frac{\partial}{\partial \tilde{\omega}}\left(\tilde{\omega}^{2} \Delta_{5} P^{*}\right)=$

$\frac{\partial}{\partial \rho}\left(\tilde{\omega}^{2} \Delta_{5} p^{\star}\right)=-j_{\theta} \tilde{\omega}$,

and

$\frac{\partial}{\partial \rho}\left(\tilde{\omega}^{2} \Delta_{5} P^{\star}\right)=-a \frac{\partial}{\partial \rho}\left(\tilde{\omega}^{2} T^{\star}\right)$.

If one writes $\tilde{\omega}^{2} \Delta_{5} P^{*}=\Omega$, then Eq. 14 can be integrated at once,

$\Omega=-\int j_{\theta}^{\tilde{\omega} \mathrm{d} p}+f(\theta)$, where $f(\theta)$ is an arbitrary function of $\theta$. It is readily checked that $f(\theta)$ is a constant or zero. Thus if $j_{\theta}$ is prescribed, $\Omega$ can be found by quadrature.

Combining Eqs. 9, 10, 11, and 14, a relation defining the unknown function is found. This is

$\frac{1}{\alpha^{2}}\left(\frac{\partial^{2} \Omega}{\partial z}+\tilde{\omega} \frac{\partial}{\partial \tilde{\omega}}\left(\frac{1}{\tilde{\omega}} \frac{\partial \Omega}{\partial \tilde{\omega}}\right)\right)+\frac{1}{2}\left(\frac{\partial \Omega}{\partial z} \frac{\partial}{\partial z}\left(\frac{1}{\alpha}\right)+\frac{\partial \Omega}{\partial \tilde{\omega}} \frac{\partial}{\partial \tilde{\omega}}\left(\frac{1}{\alpha}\right)\right)=$ -

If $\Omega$ is known from $\mathrm{Eq} .16$, then $\alpha$ may be found by solving Eq. 17. This in turn defines the toroidal component of the current, $\mathrm{J}_{\phi}$, by means of Eq. 10 , viz.,

$j_{\phi}=-\frac{\alpha \Omega}{\tilde{w}}$.

\section{A REDUCED FORCE CURRENT DISTRIBUTION}

The first two conclusions derived from the virial theorem were stated in the introduction:

1) When a system consisting of a coil and its associated magnetic field is in equilibrium, then the mechanical energy due to the electiomechanical stresses in the coil cannot be less than the magnetic energy of the field, and

2) The mechanical energy in the coil is a minimum when the coil is force-free. These conclusions lead one to intuitively guess the current distribution which will yield a force-free coil. In the past the solutions have been coils in which the field and the current slowly change from toroidal to poloidal across the windings thickness. Solutions of this nature have been investigated by Mills et al. ${ }^{8}$ and Wakefield. ${ }^{9}$ However, they lead to complicated shapes which are hard to fabricate in practice.

A poloidal surface current on a form produces a toroidal magnetic field which is completely contained inside the torus. This type of coil is subjected to an unbalanced mechanical force in the sense that the magnetic pressure acts on one side, viz., from the interior of the torus. For a purely toroidal surface current, the field is poloidal and is localized outside the torus. The direction of the mechanical forces on the torus are compressive for the toroidal current sheet and expansive for the polo:dal current sheet. Hence, a surface current 


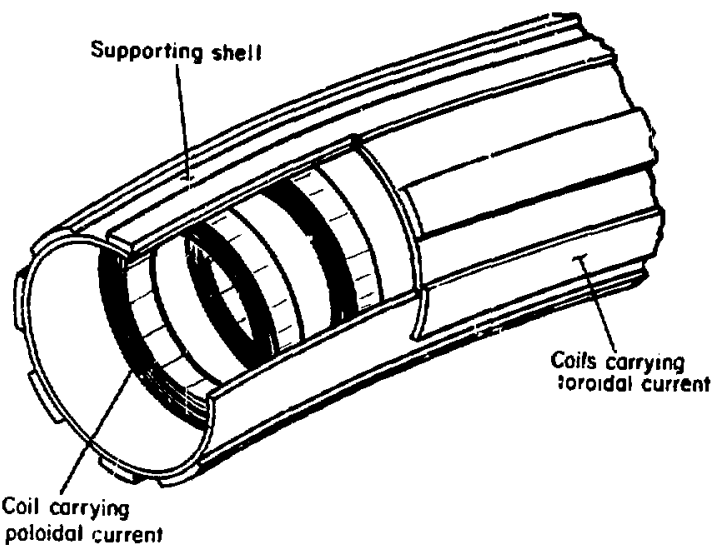

Fig. 2. Reduced force energy storage concept. The poloidal coils produce outward forces which are in opposition to the inward forces of the toroidal coils.

which has both a toroidal and a poioidal component can lead to a force-free solution, since it appears that the forces are such that they will cancel for a proper choice of current.

We therefore separate the two components of the current (toroidal and poloidal) into two distinct coils: a coil carrying a poloidal current located inside a toroidal shell, and a coil carrying a toroidal current outside the shell. A system such as this can be manufactured from circular coils.

Thus, it is a practical solution, as shown in Fig. 2. The magnitude of the currents required for the forces to nearly cancel may be obtained as follows. The poloidal current, $\mathbf{j}_{\theta}$, from

$\nabla \cdot j_{\theta}=0$,

must satisfy the condition

$j_{\theta} \tilde{\omega}=j_{\theta}^{o}(R+a)$,

where $j_{\theta}^{0}$ is the current per unit length at the rim of the torus. But, the surrent is a surface current, i.e.,

$j_{\theta}^{o}=j^{o} \delta(\rho-a)(R+a)$,

and

$j_{\theta}=j^{\circ} \delta(\rho-a) \frac{(R+a)}{(R+\rho \cos \theta)}$.
Substitution of Eq. 22 into !: 16 indicates that

$\Omega=-j^{0}(R+a)$,

inside the torus and is zero elsewhere. This property follows from the manner of taking the $1 \mathrm{init}$ of integration.

The discontinuous behavior of $\Omega$ causes the differential Equation 17 to becone

$\left(\frac{1}{\alpha}\right) \frac{R}{\rho(R+\rho \cos \theta)}+\frac{1}{2} \frac{\partial}{\partial D}\left(\frac{1}{\alpha}\right)=R+0 \cos \theta$.

The general solution of Eq. 24 is

$\alpha=\frac{(\mathrm{R}+\mathrm{a} \cos )}{\mathrm{a}} \sqrt{\mathrm{C}+\mathrm{a}^{2}}$,

where $C$ is an arbitrary constant.

The toroidal current from Eq. 18 is

$j_{\theta}=j_{0}(R+a) \frac{\sqrt{C+a^{2}}}{a} \delta(p-a)$.

We now select the value of $\mathrm{C}$ so that the magnetic forces cancel on both sides of the toroidal shell. This is equivalent to

$H_{\omega}^{2}+H_{z}^{2}=H_{\phi}^{2}$,

or from Eqs. 9, 10, and 11,

$\mathrm{j}_{\theta}^{2}=\mathrm{j}_{\phi}^{2}$

which yields $\mathrm{C}=0$, and

$j_{\theta}=j_{\phi}=j_{0}(R+a) \delta(\rho-a)$.

IV. FIGURE OF MERIT OF HINDING CONFIGLRATION The effective utilization of the superconducting material in the coil is expressed by the magnetic energy stored by the coil per unit mass of the conductor. To obtain a realistic, nelative figure of merit for the material utilization in different coils, it will be useful to compare the coils on the basis of the magnetic energy stored.

The magnetic energy stored, $E_{m}$ is given by

$E_{m}=\frac{1}{2} L I^{2}$, 
where $L$ is the inductance of the coil and $I$ is the current. But the current is related to the current density $\mathbf{j}$ by

$I=j A$,

where $A$ is the cross-sectional area of the conductor. Combining Eqs. 28 and 29, the figure of merit for the winding is

$f_{m}=\frac{1}{2} \frac{L I^{2}}{A Q^{2} D_{s}}$

where $\ell$ is the length of the superconducting winding and $\rho_{s}$ is the density of the conductor. in order to show the dependence of the figure of merit on the energy, we manipulate i.qs. 28,29 , and 30 so that

$\mathrm{fm}=\frac{\mathrm{j}}{2 a_{\mathrm{s}}} \frac{1}{\varepsilon} \sqrt{2 \mathrm{i}_{\mathrm{m}} \mathrm{L}}$.

Since the current density is nornally close to the maximum allowable value, $\frac{j}{D_{s}}$ is a constant for a given material, so that the meaning,ful parameter is $\sqrt{\frac{L}{\ell^{2}}}$.

In our case, since we have tro types of coils, the figuse of merit has to take into wecount the contrihution of the toroidal and poloidal current elements, i.e..

$f m=\frac{\sum\left(L I^{2}\right)}{2 \sum\left(A \rho_{S}\right)}$.

The indutance of a single-turn poloidal current sheet on a torus of minor radius a and major radius $R$ is given by ${ }^{2}$

$L=n_{0} R\left(1-\sqrt{\left.1-\frac{a^{2}}{R^{2}}\right)}\right.$.

However, it is known that the maximum manctic cnergy stored in a torus for a given quantity of superconductor is obtained for an aspect ratio $\frac{a}{R} \approx \frac{1}{3}$. Thus

$L_{\text {poloidal }}=0.0007183 \mathrm{R}$ (HIH),

where $R$ is expressed in centimeters.
But the value of $\ell$ for the poloidal current sheet is $2 \pi \mathrm{a}$. Thus $\frac{L}{\ell}$ ) poloidal is $\frac{0.0064647}{4 \pi^{2} R}$.

When we include the contribution of the toroidal current sheet in the force-free coil, we find

$$
\begin{aligned}
L_{\text {toroidal }} & =\mu_{0} R\left\{\left\{1+\frac{a^{2}}{8 R^{2}}\right) \log \left(\frac{8 R}{a}\right)-\frac{\pi}{4}+\frac{a^{2}}{24 R^{2}}\right\} \\
& =0.01849 R \quad \text { (HH) }
\end{aligned}
$$

where we have assumed $\frac{a}{R}=1 / 3$. Since the appropriate value of $\ell$ for the toroidal current sheet is $2 \pi R$, the quantity $\frac{L}{\ell^{2} \text { tornidal }}$ is $\frac{0.01849}{4 \pi^{2}}$.

Now we note that

$l_{m}$ )toroidal/E Jpoloidal $=L^{2} I^{2}$ toroidal $/ L I^{2}$, poloidal

Since the calculations of section 111 indicated that the current Jensities in the toroidal and poloidal coils must be equal in order to satisfy the force-free condition, we find

$$
\begin{aligned}
\frac{\left.E_{m}\right)_{\text {toroidal }}}{E_{m}{ }_{\text {poloidal }}} & =\frac{(0.01849 \mathrm{R})}{(0.000718 \mathrm{R})} \frac{\mathrm{a}^{2}}{\mathrm{R}^{2}} \\
& =2.8613 .
\end{aligned}
$$

or

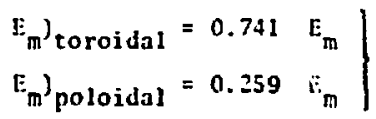

where $E_{m}=E_{m}{ }^{\prime}$ toroidal $\left.+E_{m}\right)_{p o l o i d a l}$ is the total energy stored in the coil. The expression for the figure of merit for the force-free coil becomes

$$
\begin{aligned}
f m & =\frac{i}{\rho_{s}} \sqrt{\frac{E_{m}}{8 \pi^{2} R}} \frac{\sqrt{0.741 L_{\text {toroidal }}}+\frac{a}{R} \sqrt{0.2591_{\text {poloidal }}}}{1+\left(\frac{a}{R}\right)^{2}} \\
& =\frac{0.111 j}{D_{s}} \sqrt{\frac{E_{m}}{8 \pi^{2} R}} .
\end{aligned}
$$

We now compare the $f m$ of other coils with that of the foice-free co:l discussed here.

The first coil we consider is a cylindrical coil of radius $a_{0}$ and lergth $\ell_{0^{*}}$ Nagaoka ${ }^{10}$ evaluated 
the inductance of a cylindrical curreni sheet which is given by

$t=0.002 \pi^{2} a_{0}\left(\frac{2 a_{0}}{l_{0}}\right) K(\omega t)$.

where the Vagaoka factor, $K$, is a function of the ratio $\frac{2 z_{0}}{t_{0}}$, and all dimensions are expressed in centinet ers.

Because of the idealized situation assumed here, $v i z$, that of a very thin toil, the maimum inductance is not that for a Brooks coill, int insteat ${ }^{12}$ it is for $\frac{2 a}{k_{0}}=2.4510$. The corresponding induct ance is

$L=0.02305 \mathrm{a}_{0}(2.11)$

The figure of morit for the cylintritul arrani

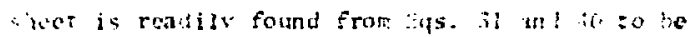

$t i=0.152 \frac{i}{s_{s}} \quad \frac{1 n_{m}}{s-a_{0}}$

llat we considereal the poloisal elenont alone. as is the case for the conventional taroidal coil. then the figure of nerit would ho

$f m=0.011 \frac{j}{s} \quad \frac{i}{s-m_{R}}$.

The above calculations show clearly that in 'he ferce-frec coil the material is used more efficicntIy than in a conventional toroidal winding. The figure of merit for a force-free coil is intermeliate between that of a coil with pure poloidal current and that of a coil aith pure toroidal current. The use of a superconluctor is thus slizntly less efficient than in a solenoid; however, this is more than compensated for by the economy of the mechanical structure.

v. COIJ. REI:IFORCINC STRUICTIRE

The ahove calculations for the current distributions needed to ohtain a forc.:-free coil were performed on the basis of the cirrent being concent ratud on a surface (sheet). Actually, a toroidal form i inceded to support the tho sets of coils which carry the toroidal and poloidal itrocuts. The two coils are therefore separated by a distance eyal to the thickitess of the form. is a result the tores are almost bat not quite cancelled.

Before we estinate this imbalance. he first noed to evaluate the thichness of the toroidal shell required to sustain the foress. Irot: the virial theorem, anc obtaines

$\varphi=\frac{i s}{i} i_{n}$

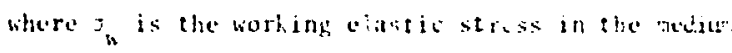

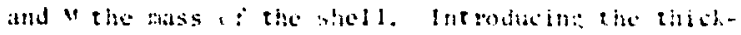

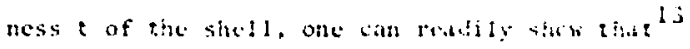

$t=1 \frac{i^{2}}{2 \ldots 6 n}+\frac{1}{2 n} k$.

$\operatorname{sincos} \frac{a}{R}=\frac{1}{j}$, and ior typical salues $b=0.005 \mathrm{~T}$

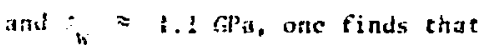

$\frac{1}{R}=\frac{1}{600}$

which indicates that the thin-shell theoty is valion In imadiate somseliatence of thas cheory is that one can estimate the effect of imhalince in the nainet is forces.

In the forcenfec situation, the shell is abler purc eompresion ffron the inside and iros tite but side). If there is an iribalance dac to the tinite thichnes: of the shell, we expert to have a difforence in the magnetic ficld, $\therefore B_{2}$ of the order $t / R$.

There are therefore additional st resses, in tl:c forn of hoop stresses caused by this net force. The

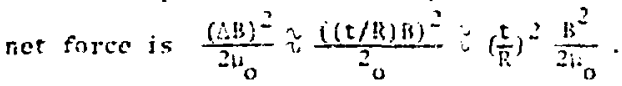
The hoop stresses (in direction tangential io the minor and mijor circles) are,

$$
\begin{aligned}
\sigma & =\frac{2 \pi}{3}\left(\frac{\Delta B^{2}}{2 H_{0}}\right) \frac{R}{t}(1+a / R) \\
& =8.377 \frac{t}{R}\left(\frac{B^{2}}{2 \mu_{0}}\right) .
\end{aligned}
$$


The stres'es are increased by approximately $1 / 75$ of the normal worting stresses.

Ar essential conclusion is that for the forcefrew case, the rass defined $b y$ i:q. 43 is alout $1 / 3$ that for the mass needed in a conventional coi?. indeed ftelly" hats shown that fot a regular toil $43.1 \frac{3}{35} 5$. 1.9

\section{SYSTI GOSTS}

The cost of the Superconducting lagnet ic Ferge storar system is ofitained by the frocedure outlined iy lassenzahl at $4 !^{1.4}$ the cost of the system will tepend on the costs of

1) the sunereondact or miterial

$\therefore$ the dewar

it the st ructure

1) the interfacing cquipment (ac-de converters]

5) nistellancous.

\section{Supezconductor haterial}

since two sets of bolls are used, one would he terated to st te that the cost of the material should ne doubled. Ketualin, the increase is less beciuse the inductance of the force-free coil is sreater that that of a conventional to roidal coil.

If the inductance of a solonotdal coil is $t_{1}$ and that of the adutional toroidal winding is $L_{\text {... }}$ then the chergy stured in a conventional coil is

$1_{\mathrm{mi}}=\sum_{i}^{1} \mathrm{i}_{\mathrm{i}} \mathrm{l}_{\mathrm{i}}: \quad \mathrm{i}=1, \ldots$.

On the otine hand, for the foree-frec coil consisting af thiti toroinal and solonosial coils,

$r_{r 1}=\frac{1}{2} r^{2}\left(r_{1}+r_{2}\right)$

To determine the inductance of a force-free coil, which will store the sante energy as that stored in a convertional solenoidal coil, we must iave

$1 \frac{2}{1} L_{1}=1_{2}^{2}\left(L_{1}+L_{2}\right)$

The current, therefore, must be reduced by the ratio

$\frac{I_{1}}{T_{2}}=\left(\frac{L_{1}+L_{2}}{L_{1}}\right)^{I / 2}=\left(I+\frac{L_{2}}{L_{1}}\right)^{1 / 2}$.
If we keep the current the same, the inductance of the comparison coil must be increased by the factor $\left(1+\frac{L_{2}}{L_{1}}\right)$. Since the inductance is proportional to the square of the number of turns, the mass of the superconductor would vary like the square root of the inductance.

Therefore, we conclude that instead of doubling tw. mass of superconductor, we have indeed increased it by $1+\left(\frac{L_{1}}{L_{2}}\right)^{1 / 2}$. Using the values of Eạs. 36 and 40 , we find that the factor 151.18 (i.e., an inc rease of $18^{\circ}$ ).

The cost of the dewar is the sanc as for a simple toroidial coil.

The cost of the support structure, however, is roluced appreciably. lie had mentioned above that Stekly predicted the mass of a conventional coil wounis or a torus to be $\mathbf{3} .1$ times bigger than that of ine risimum mass. This factor cones close to the raluc of 3.0 found by Hassenzahl. ${ }^{\text {th }}$ For the force-free coil, it is taken to be equal to the minimum value. The cost estimates given for a force-free coil in Table 1 are based on $B_{\max }=3.0 \mathrm{~T}$ and $\mathrm{a} / \mathrm{R}=1 / 3$. These costs are compared to the costs of some conrentional roils in Table II.

TABLE I

COST ESTIMATES FOR FORCE-FREE COIL

\begin{tabular}{|c|c|c|c|c|}
\hline Energy Stored (MJ) & $3 \times 10^{4}$ & $10^{6}$ & $3.9 \times 10^{7}$ & $10^{8}$ \\
\hline $\begin{array}{l}\text { Cost of supercoiduct or } \\
\left(5 \times 10^{6}\right)\end{array}$ & 2.24 & 22.77 & 261.96 & 489.7 \\
\hline Dewar cost $\left(\$ \times 10^{5}\right)$ & 2.0 & 24.6 & 300 & 581 \\
\hline Structure $\left(\$ \times 10^{6}\right)$ & 0.267 & 8.67 & $3 \overline{38}$ & 866.67 \\
\hline $\begin{array}{c}\text { ac-de converter } \\
\left(S \times 10^{6}\right)\end{array}$ & 0.04 & 1.4 & 54 & 140 \\
\hline Miscellaneous $\left(\$ \times 10^{6}\right)$ & .47 & 2.1 & 51 & 128 \\
\hline TOTAL & 5.02 & 59.54 & 1004.96 & 2205.37 \\
\hline
\end{tabular}

TABLE II

COMPARISON OF CONSTRUCTION COSTS OF FORCE-FREE CUIL WITH COSTS OF CYLINDRICAL COIL

$\begin{array}{lccll}\text { Energy (MI) } & 3 \times 10^{4} & 10^{6} & 3.9 \times 10^{7} & 10^{8} \\ \text { Force-free coil }\left(\$ 10^{6}\right) & 5.02 & 59.54 & 1004.96 & 2205.37 \\ \text { Conventional coil }\left(\$ 10^{6}\right) & 5.2 & 73.4 & 1641 & 3864\end{array}$

This table clearly demonstrates the economic advantage of the force-free coil. 


\section{ACKNOWLCDGNENTS}

It is a pleasure to acknowledge the hospitality of W. E. Keller and W. V. Hassenzahl which made it possible for the author to complete this study during a brief visit to Los Alamos. The author also wishes to axpress his appreciation for the many stimulating discussions he had with $W$. V. Hassenzahl.

\section{REFERENCES}

1. R. W. Boom and H. A. Peterson, "Superconducting Energy Storage for Fower Systems," IEEE Trans. Magn. MAG-8, 701-703 (1972).

2. Z. J. J. Stekly, "Magnetic Energy Storage Using Superconducting Coils," AVCO-Everett Research Laboratory report AMP-102, Everett, MA (1963).

3. R. Lüst and A. Schlüter, "Kraftfreie Magnetfelder," $Z$. Astrophys. 34, 263-282 (1954).

4. H. P. Furth, M. A. Levine, and R. W. Haniek, "Production and Use of High Transient Magnetic Fields. II," Rev. Sci. Instrum. 28, 949-958 (1957).

5. C. L. Longmi re, Elementary Plasma Physics (WileyInterscience Publicarions, New York, 1963).

6. M. S. Lubell, H. M. Long, J. N. Luton, and W. C. T. Stoddart, "Economics of Large Superconducting, Toroidal Magnets for Fusion Reactors," Oak Ridge National Laboratory report ORNL-TM-3927 (1972),
7. S. Chandrasekhar, "On Force-Free Magnetic fields," Proc. Nat. Acad. Sci. U.S.A. 42, 1-5 (1956).

8. R. J. Mills, D. R. helis, A. Bahr and R. L. Peshin, "Design of a Reduced Force Coil Systua," Plasma Physics Laboratory memorandum MATT-111, Princeton University, Princeton, is (1960).

9. K. E. Wakefield, "Design of Force-Free Toroidat Magnets," Plasma Physics l.aboratory memorandun MATT-208, Princeton University, Princeton, NJ (1964).

10. Nagaoka, J. Col1. Sci, Tohyo 27, IE (1YUy).

11. H. B. Brooks, "Design of Standards of Inductance and the Proposed Use of Model Reactors in tire Design of A.ir-Core and Iron-Core Reactors." $J$. Res. Nat. Fur. Stand. 7, 289-328 (1931).

12. F. W. Grover, Inductance Calculations: riorking Formulas and Tables (Dover Publicutions, New York, 1952\}.

13. A. E. Love, Elastility, (Dover Publications, New York, 1944).

14. W. V. Hassenzahl, B. L. Baker, and W. L. Keller, 'The Economics of Superconducting Magnetic Energy Storage Systems for Load Leveling: A Conparison with other Systems," Los Alamos Scientific Laboratory report LA-5377-AS (1973).

\section{APPENDIX}

PROOF OF VIRIAL. THEOREM

The virial theorem of Ciausius can be extended by analogy to the case of a coil and its magnetic field. It is known from classical electromagnetic theory that Maxwe1l's stress tensor $\stackrel{一}{\doteq}$ satisfies a conservation relation of the form

$\frac{\partial}{\partial x_{j}}\left(T_{i j}\right)=0$.

When the electromagnetic forces are in equilibrium with elastic forces in a medium that contains the electromagnetic field, then through the introduction of an elastic stress tensor $\underline{\underline{S}}$, the above equation is modified to become

$\frac{\partial}{\partial x_{j}}\left(T_{i j}+s_{i j}\right)=0$.

If we multiply the above expression by the position vector $\underline{r}$ and integrate over all space

$$
\begin{aligned}
0 & =\int_{v} x_{i} \frac{\partial}{\partial x_{j}}\left(T_{i j}+s_{i j}\right) d \tau \\
& =\int_{s} x_{i}\left(T_{i i}+s_{i i}\right) n_{i} d \sigma-\int_{v}\left(T_{i i}+s_{i i}\right) d \tau
\end{aligned}
$$

In the above equation, $\mathrm{d} \tau$ is an e'ement of volume, do an element of surface, the $n_{i}$ 's are the direction cosines of the cutward normal to the surface $S$ and the transition from the first to the second line of Eq. A- 3 has made use of Gauss's theorem. Since the trace of the tensor $I$ is the magnetic energy, while the trace of $\underline{\underline{S}}$ is the elastic energy, one finds at once

$\int\left(\frac{\mathrm{B}^{2}}{2 \mu_{0}}+\frac{1}{2} \frac{\sigma^{2}}{\varepsilon}\right) \mathrm{d} \tau=\int \mathrm{x}_{\mathrm{i}}\left(\mathrm{T}_{\mathrm{i}}+\mathrm{S}_{\mathrm{ii}}\right) \mathrm{n}_{\mathrm{i}} \mathrm{d} \sigma$.

The above relation is the virial theorem. 
If the coil is of infinite dimension. the surface integral vanishes, and the rechanical enerzy is equal to the magnetic energy except for a negative sipn. The nepative sign indicates that the elastic forces have to be tensile in chatacter.

when the coil is of finite dinensions. The surface jntegral need not vinish. Hut becuse of the positive definite character of the integrand $\int \frac{\theta^{2}}{2 \mu_{0}} d \tau \leq\left(-\int \frac{1}{2} \frac{q^{2}}{E} d \tau\right)$

lt is thus clear that the clastic energy stored in the coil is at least as smali as the magnetic energy but is usually larger. In a force-free coil the mechanical energ is a minimum, i.c., $E_{i n}=$ Mechanical energy. 